\title{
NECESSARY CONDITIONS FOR EMBEDDING RINGS INTO FIELDS $\left({ }^{1}\right)$
}

\begin{abstract}
BY
ABRAHAM A. KLEIN

1. Introduction. If a (noncommutative) integral domain $R$ is embeddable in a (skew-) field $D$, then the multiplicative semigroup $R^{*}=R-\{0\}$ is embeddable in the group $D^{*}=D-\{0\}$. Mal'cev [6] has constructed an integral domain $R$ whose multiplicative semigroup $R^{*}$ cannot be embedded in a group and hence the ring $R$ cannot be embedded in a field. In [4], we have constructed integral domains that cannot be embedded in fields, but their multiplicative semigroups are embeddable in groups. Our construction was based on the following property of matrix rings over fields:
\end{abstract}

$$
\mathfrak{N}_{l} \text { : If a matrix } C \text { of order } 1 \text { is nilpotent, then } C^{l}=0 .
$$

Clearly, this condition holds also in subrings of fields, and it is therefore necessary for embedding a ring in a field. We have constructed integral domains $R$ which do not satisfy $\mathfrak{N}_{l}$, for some $l \geqq 3$, but the semigroups $R^{*}$ satisfy a certain sufficient condition for embeddability in a group.

The problem arises: What is the relation between the necessary conditions $\mathfrak{R}_{l}, l=1,2, \ldots$ and the trivial necessary condition

\section{$\mathfrak{M}: R^{*}$ is embeddable in a group.}

The aim of this paper is to prove that if an integral domain satisfies all the conditions $\mathfrak{N}_{l}$, then it also satisfies $\mathfrak{M}$. Thus, if we denote the condition $\bigcap_{l=1}^{\infty} \mathfrak{N}$ l by $\mathfrak{N}$ and we restrict ourselves to integral domains, we have: $\mathfrak{N} \subseteq \mathfrak{M}$. Moreover, since by [4] there exist integral domains which satisfy $\mathfrak{M}$, but do not satisfy $\mathfrak{R}_{l} \supseteq \mathfrak{R}$ (for some $l \geqq 3$ ) we obtain: $\mathfrak{N} \varsubsetneqq \mathfrak{M}$. The problem whether the condition $\mathfrak{N}$ is sufficient for an integral domain to be embeddable in a field remains open.

Our main result is achieved by showing that if $R$ is an integral domain and satisfies $\mathfrak{R}$, then the semigroup $R^{*}$ satisfies all the necessary and sufficient conditions of Mal'cev [7] for embeddability in a group. Our formulation of Mal'cev's conditions is a slight modification of that given by Cohn [1]. We show that for cancellative semigroups, a restricted set of conditions, which we call "cancelled conditions" is sufficient for embeddability. We prove that the cancelled conditions have a certain structure and using it we obtain that $\mathfrak{R}_{l}$ implies all the cancelled conditions of order

Received by the editors June 2, 1967 and, in revised form, December 10, 1967.

( $\left.{ }^{1}\right)$ This is a part of the author's Ph.D. thesis prepared at the Hebrew University of Jerusalem under the kind supervision of Professor S. A. Amitsur. 
$l$ in $R^{*}$. Hence $\mathfrak{N}=\bigcap_{l=1}^{\infty} \mathfrak{R}_{l}$ implies that all the cancelled conditions hold in $R^{*}$ and therefore $R^{*}$ is embeddable in a group.

In the last section of the paper we study the relation between $\mathfrak{N}_{l}$ and the condition

\section{$\mathfrak{M}_{l}: R^{*}$ satisfies all the conditions of Mal'cev of order $l$.}

The result is $\mathfrak{R}_{1}=\mathfrak{M}_{1}, \mathfrak{R}_{2}=\mathfrak{M}_{2}$ and $\mathfrak{R}_{l} \varsubsetneqq \mathfrak{M}_{l}$ for $l \geqq 3$.

At the end of the paper we give an application to our main result and some remarks about the condition $\mathfrak{N}$ which have been pointed out by the referee.

2. Mal'cev's conditions. As in [1] we obtain Mal'cev's conditions by the aid of finite chains of brackets of two kinds, round (, ) and square [, ], which are labelled by suffixes. We need only "normal" chains in the sense of

Definition 1. A chain of brackets will be called normal if the round brackets occur in the chain in pairs of the form

$$
\cdots\left({ }_{i} \cdots\right)_{i} \cdots
$$

$i=1, \ldots, r$, and if $1 \leqq i<i^{\prime} \leqq r$, then $\left({ }_{i}\right.$, appears in the chain after $\left({ }_{i}\right.$, and if it appears before $)_{i}$ then $)_{i}$, appears also before $)_{i}$. Similar conditions are imposed on the square brackets.

We shall say that a normal chain is of order $l$ if it contains $r$ pairs of round brackets and $s=l-r$ pairs of square brackets.

Using a normal chain of order $l$ we obtain a condition of Mal'cev of order $l$ in the following way:

For each $i, i=1, \ldots, r$ we adjoin 4 elements (indeterminates) $a_{i}, p_{i}, b_{i}, b_{i}^{\prime}$ to the $i$ th pair of round brackets, and in the chain we insert the words $a_{i} b_{i}$ before $\left(_{i}, p_{i} b_{i}\right.$ after it; $p_{i} b_{i}^{\prime}$ before $)_{i}, a_{i} b_{i}^{\prime}$ after it and obtain

$$
\cdots a_{i} b_{i}\left({ }_{i} p_{i} b_{i} \cdots p_{i} b_{i}^{\prime}\right)_{i} a_{i} b_{i}^{\prime} \cdots
$$

Similarly for each $j, j=1, \ldots, s$, we adjoin 4 elements $d_{j}, q_{j}, c_{j}, c_{j}^{\prime}$ to the $j$ th pair of square brackets, and in the chain we insert $c_{j} d_{j}$ before $\left[{ }_{j}, c_{j} q_{j}\right.$ after it; $c_{j}^{\prime} q_{j}$ before ]$_{j}, c_{j}^{\prime} d_{j}$ after it and obtain

$$
\cdots c_{j} d_{j}\left[c_{j} q_{j} \cdots c_{j}^{\prime} q_{j}\right]_{j} c_{j}^{\prime} d_{j} \cdots
$$

Now, we have two words between any two brackets of the chain and we equate them. Since the chain contains $2 l$ brackets we obtain $2 l-1$ equations which will be called the internal equations of the chain. Equating the left word of the first bracket with the right word of the last bracket, we obtain an equation which will be called the external equation of the chain.

The condition of $\mathrm{Mal}^{\prime} \mathrm{cev}$ corresponding to the above chain is: If for any $4 l$ elements of the semigroup $a_{i}, p_{i}, b_{i}, b_{i}^{\prime}, d_{j}, q_{j}, c_{j}, c_{j}^{\prime}, i=1, \ldots, r, j=1, \ldots, s$, the $2 l-1$ internal equations are satisfied, then the external equation is also satisfied.

Mal'cev's theorem is: A semigroup with 1 is embeddable in a group if and 
only if it satisfies the conditions of Mal'cev corresponding to all normal chains [1, p. 268].

In the $4 l$ words of two letters, which we have inserted in a normal chain of order $l$, the elements $a_{i}, p_{i}, c_{j}, c_{j}^{\prime}$ occur as left terms and will be called left elements; the elements $d_{j}, q_{j}, b_{i}, b_{i}^{\prime}$ occur as right terms and will be called right elements.

From now on, we shall deal with cancellative semigroups since we are interested in the multiplicative semigroups $R^{*}$ of an integral domain which clearly satisfies the cancellation laws. For such semigroups we shall show that a restricted set of conditions which we call "cancelled conditions" is sufficient for embeddability in a group.

Definition 2. A chain will be called cancelled if it is normal and for each $i, i=1, \ldots, r$, the bracket $\left({ }_{i} \text { is not followed by }\right)_{i}$ and if $\left({ }_{1}\right.$ is the first bracket of the chain then $)_{1}$ is not the last; similar conditions are imposed on the square brackets. A condition of Mal'cev is called cancelled if it corresponds to a cancelled normal chain.

LEMMA 1. If a cancellative semigroup $S$ satisfies all the cancelled conditions, then it is embeddable in a group.

Proof. The conditions corresponding to the chains of order 1 are implied by the cancellation laws. Again using the cancellation laws it is easily shown that each noncancelled condition of order $l$ follows from a condition of order $l-1$, and by induction we obtain that $S$ satisfies all the conditions of Mal'cev. Hence by Mal'cev's theorem if $1 \in S$ then $S$ is embeddable in a group. If $1 \notin S$, embed $S$ in $S_{1}=S \cup\{1\}$ and observe that any chain of equations in $S_{1}$ becomes a chain in $S$ on multiplying by an element $t \in S$ (which can again be cancelled) from left and right.

3. The structure of the cancelled conditions. Let us fix a cancelled condition of order $l$ and suppose it contains $r$ pairs of round brackets and $s=l-r$ pairs of square brackets. By Definition 2 , it follows that $r \geqq 1$ and $s \geqq 1$. Consider the corresponding cancelled condition which is obtained, as in the previous section, by the aid of $4 l$ elements $a_{i}, p_{i}, b_{i}, b_{i}^{\prime}, d_{j}, q_{j}, c_{j}, c_{j}^{\prime}, i=1, \ldots, r, j=1, \ldots, s$. Again by Definition 2, it follows that the two left (right) elements of the words of an equation of the condition are distinct. Hence each element appears in exactly two equations.

For two left elements $u, v$ define $u \sim v$ if there exist left elements $u=u_{0}, u_{1}, \ldots, u_{k}$, $u_{k+1}=v$ and $k+1$ equations of the condition as follows

$$
u_{0} x_{0}=u_{1} y_{1}, \quad u_{1} x_{1}=u_{2} y_{2}, \ldots, \quad u_{k} x_{k}=u_{k+1} y_{k+1} .
$$

It is easily verified that " $\sim$ " is an equivalence relation in the set of left elements and we obtain a partition of this set into disjoint classes. A similar process leads to a partition of the set of right elements into disjoint classes. The class which is determined by an element $z$ will be denoted by $\bar{z}$.

LEMMA 2. If in (1) $u_{k+1}=u_{0}$ then $\bar{u}_{0}=\left\{u_{0}, u_{1}, \ldots, u_{k}\right\}$. 
Proof. Clearly $\bar{u}_{0} \supseteq\left\{u_{0}, u_{1}, \ldots, u_{k}\right\}$. Let $w \in \bar{u}_{0}$ then $u_{0} \sim w$ and since each element is contained in exactly two equations we have either

$$
u_{0} x_{0}=u_{1} y_{1}, \quad u_{1} x_{1}=u_{2} y_{2}, \ldots, u_{i} x_{i}=w y,
$$

or

$$
u_{0} y_{k+1}=u_{k} x_{k}, \quad u_{k} y_{k}=u_{k-1} x_{k-1}, \ldots, u_{j} y_{j}=w x .
$$

In both cases $w$ belongs to $\left\{u_{0}, u_{1}, \ldots, u_{k}\right\}$ and the result follows.

LEMma 3. For $i=1, \ldots, r$ all the elements of the class $\bar{p}_{i}$ are contained in the equations which appear in the interval $\left({ }_{i} \cdots\right)_{i}$ of the chain.

Proof. For each square bracket the words before and after it have the same left-hand factor. For any round bracket occuring in the interval $\left({ }_{i} \ldots\right)_{i}$ its mate also occurs; this shows that all left-hand factors in the interval $\left({ }_{i} \ldots\right)_{i}$ occur in pairs and the result follows from this.

Going through the proof of Lemma 3 we obtain

LEMMA 4. (a) If $a_{i^{\prime}} \in \bar{p}_{i}$, then $i^{\prime}>i$, and in particular $a_{1} \notin \bar{p}_{i}$ for all $i=1, \ldots, r$.

(b) If $\left({ }_{i+1} \cdots\right)_{i+1}$ is contained in $\left({ }_{i} \cdots\right)_{i}$, then $a_{i+1} \in \bar{p}_{i}$.

(c) If $i^{\prime} \neq i$, then $\bar{p}_{i^{\prime}} \cap \bar{p}_{i}=\varnothing$.

The analog of Lemma 4 for the right elements is

LeMma $4^{\prime}$. (a) If $d_{j^{\prime}} \in \bar{q}_{j}$, then $j^{\prime}>j$, and in particular $d_{1} \notin \bar{q}_{j}$ for all $j=1, \ldots, s$.

(b) If $\left[j_{j+1} \cdots\right]_{j+1}$ is contained in $\left[{ }_{j} \cdots\right]_{j}$, then $d_{j+1} \in \bar{q}_{j}$.

(c) If $j^{\prime} \neq j$, then $\bar{q}_{j^{\prime}} \cap \bar{q}_{j}=\varnothing$.

The structure of our cancelled condition is given by

THEOREM 5. The set of left elements consist of $r+1$ classes which are $\bar{a}_{1}, \bar{p}_{1}, \ldots, \bar{p}_{r}$, and the set of right elements consists of $s+1$ classes which are $\bar{d}_{1}, \bar{q}_{1}, \ldots, \bar{q}_{s}$.

Proof. We prove the theorem for the left elements and in a similar way it can be proved for the right elements.

By (a) and (c) of Lemma 4, the classes $\bar{a}_{1}, \bar{p}_{1}, \ldots, \bar{p}_{r}$ are disjoint. It remains to show that all the left elements $a_{i}, p_{i}, c_{j}, c_{j}^{\prime}, i=1, \ldots, r, j=1, \ldots, s$ belong to one of these classes.

The assertion is clear for $p_{1}, \ldots, p_{r}$ and let us prove it for $a_{i}, i=1, \ldots, r$. For $i=1$ we have $a_{1} \in \bar{a}_{1}$. Let $i \geqq 2$ and assume that the assertion is true for $i^{\prime}, i^{\prime}<i$. We consider two cases:

(a) $\left({ }_{i} \cdots\right)_{i}$ is contained in $\left(i_{-1} \cdots\right)_{i-1}$. Then by Lemma 4(b) with $i-1$ replacing $i$ we obtain $a_{i} \in \bar{p}_{i-1}$.

(b) $\left(_{i} \text { appears after }\right)_{i-1}$. Then the interval $)_{i-1} \cdots\left({ }_{i}\right.$ does not contain round brackets of the form (. Let $)_{i}$, be the last round bracket of this interval and $i^{\prime}=i-1$ if this interval does not contain round brackets. We have $i^{\prime}<i$ and considering the interval $)_{i} \cdots\left({ }_{i}\right.$ we obtain that $a_{i}$ and $a_{i}$ belong to the same class. By the induction 
hypothesis $a_{i}$, belongs to one of the classes $\bar{a}_{1}, \bar{p}_{1}, \ldots, \bar{p}_{r}$ and hence $a_{i}$ also belongs to one of these classes.

It remains to prove that each of the elements $c_{j}, c_{j}^{\prime}, j=1, \ldots, s$ belongs to one of the classes $\bar{a}_{1}, \bar{p}_{1}, \ldots, \bar{p}_{r}$.

For $c_{j}$ consider the nearest round bracket to ${ }_{j}$ and let it belong to the $i$ th pair. We obtain either $c_{j} \in \bar{p}_{i}$ or $c_{j} \in \bar{a}_{i}$ and we have already proved that $\bar{a}_{i}$ is one of the classes $\bar{a}_{1}, \bar{p}_{1}, \ldots, \bar{p}_{r}$. In a similar way we obtain the result for $c_{j}^{\prime}$ which completes the proof of the theorem.

The following result will be used in the next section.

LEMMA 6. If ${ }_{1}$ is the first bracket of the chain, then $b_{1} \in \bar{d}_{1}$.

Proof. The first square bracket of the chain is $\left[_{1}\right.$ and the interval $\left(_{1} \cdots\left[_{1}\right.\right.$ is either empty or contains round brackets of the form (. If it is empty then $p_{1} b_{1}=c_{1} d_{1}$ and hence $b_{1} \in \bar{d}_{1}$. In the other case we have for some $i>1$ :

$$
p_{1} b_{1}=a_{2} b_{2}, \ldots, p_{i-1} b_{i-1}=a_{i} b_{i}, p_{i} b_{i}=c_{1} d_{1} .
$$

Hence again we obtain $b_{1} \in \bar{d}_{1}$.

4. The main result. If $R$ is a ring which satisfies the condition $\mathfrak{R}_{l}$ and if $A, B \in R_{l}$ and $(A B)^{l}=0$ then $(B A)^{l}=0$. Indeed, $B A$ is nilpotent since $(B A)^{l+1}=B(A B)^{l} A=0$, and therefore $(B A)^{l}=0$ since $R$ satisfies $\mathfrak{R}_{l}$. Using this remark and the results of the previous sections we shall obtain the main result of this paper which is:

THEOREM 7. If $R$ is an integral domain which satisfies the condition $\mathfrak{R}=\bigcap_{l=1}^{\infty} \mathfrak{R}_{l}$, then $R^{*}$ is embeddable in a group, i.e., $\mathfrak{R} \subseteq \mathfrak{M}$.

Proof. By Lemma 1 it suffices to prove that $R^{*}$ satisfies all the cancelled conditions.

Let us consider the cancelled condition of the previous section and assume that for $4 l$ elements of $R^{*}$

$$
a_{i}, p_{i}, b_{i}, b_{i}^{\prime}, d_{j}, q_{j}, c_{j}, c_{j}^{\prime}, \quad i=1, \ldots, r, j=1, \ldots, s,
$$

the internal equations hold and we have to prove that the external equation holds. Assume that ${ }_{1}$ is the first bracket of the chain and a similar proof can be given in the other case. Denote by ef the right word of the last bracket of the chain and let $w=a_{1} b_{1}-e f$. The external equation is $a_{1} b_{1}=e f$ and hence the result will follow if we prove that $w=0$.

Using the $4 l$ elements of (2) and 0 we first construct two matrices $A_{1}, B_{1}$ of orders $(r+1) \times l$ and $l \times(s+1)$ respectively. Then by the aid of 0 and an element $t \neq 0$ we shall enlarge $A_{1}, B_{1}$ to $A, B \in R_{l}$ which will serve to obtain the result.

The two words appearing near each bracket of the given chain have one common letter: $b_{i}, b_{i}^{\prime}, c_{j}, c_{j}^{\prime}$ for $\left(_{i},\right)_{i},[j,]_{j}$ respectively. We put a minus sign before the elements which are common to the words of the $2 k$ th bracket of the chain, $k=1,2$, $\ldots, l$. Note that since $\left(_{1}\right.$ is the first bracket of the chain " - " is not adjoined to $b_{1}$. 
$A_{1}$ is obtained by the aid of the $r+1$ classes of left elements (Theorem 5) and zeros. We put the elements of $\bar{a}_{1}$ (with the adjoined signs) in the first row, the elements of $\bar{p}_{1}$ in the second row, $\ldots$, the elements of $\bar{p}_{r}$ in the $(r+1)$ th row. The place of each element in the row of its class will be determined by the table:

\begin{tabular}{|c|c|c|c|c|c|}
\hline 1 & $\ldots$ & $r$ & $r+1$ & $\ldots$ & $l$ \\
\hline \hline$a_{1}$ & $\ldots$ & $a_{r}$ & $\pm c_{s}$ & $\ldots$ & $\pm c_{1}$ \\
\hline$p_{1}$ & $\ldots$ & $p_{r}$ & $\pm c_{s}^{\prime}$ & $\ldots$ & $\pm c_{1}^{\prime}$ \\
\hline
\end{tabular}

We put zeros in the remaining places.

$B_{1}$ is obtained by the aid of the $s+1$ classes of right elements and zeros. We put the elements of $\bar{q}_{s}$ in the first column,..., the elements of $\bar{q}_{1}$ in the sth column and those of $\bar{d}_{1}$ in the $(s+1)$ th column. The place of each element in the column of its class will be determined by the table:

\begin{tabular}{|l||c|c|}
\hline 1 & $b_{1}$ & $\pm b_{1}^{\prime}$ \\
\hline$\vdots$ & $\vdots$ & $\vdots$ \\
\hline$r$ & $\pm b_{r}$ & $\pm b_{r}^{\prime}$ \\
\hline$r+1$ & $d_{s}$ & $q_{s}$ \\
\hline$\vdots$ & $\vdots$ & $\vdots$ \\
\hline$l$ & $d_{1}$ & $q_{1}$ \\
\hline
\end{tabular}

As in $A_{1}$ the remaining places are filled in by zeros.

By Lemma 4(a) if $a_{i} \in \bar{p}_{i}$ then $i^{\prime}>i$. Whence $a_{i}$ appears in $A_{1}$ on the right side of $p_{i}$ and this implies that on the left side of $p_{i}$ appear only zeros. Similarly, by Lemma $4^{\prime}(a)$ we obtain that in $B_{1}$ appear only zeros on the left side of $q_{j}$. By Lemma $6 b_{1} \in \bar{d}_{1}$ and hence $b_{1}$ appears in the last column of $B_{1}$.

The general element of $A_{1} B_{1}$ is a sum whose $i$ th term, $1 \leqq i \leqq r$, is 0 or one of the words $\pm a_{i} b_{i}, \pm p_{i} b_{i}, \pm p_{i} b_{i}^{\prime}, \pm a_{i} b_{i}^{\prime}$ and whose $(r+s+1-j)$ th term, $1 \leqq j \leqq s$, is 0 or one of the words $\pm c_{j} d_{j}, \pm c_{j} q_{j}, \pm c_{j}^{\prime} q_{j}, \pm c_{j}^{\prime} d_{j}$.

Each of the words

$$
a_{i} b_{i}, p_{i} b_{i}, p_{i} b_{i}^{\prime}, a_{i} b_{i}^{\prime}, c_{j} d_{j}, c_{j} q_{j}, c_{j}^{\prime} q_{j}, c_{j}^{\prime} d_{j}, \quad i=1, \ldots, r, \quad j=1, \ldots, s
$$

perhaps with " - " is obtained as a summand of one and only one element of $A_{1} B_{1}$. 
If $x y$ and $u v$ are words of (3) and $x y=u v$ is one of the $2 l$ equations of the condition, then $x, u$ belong to the same left class, and $y, v$ belong to the same right class. Hence $\pm x, \pm u$ are in the same row of $A_{1}$, say the $m$ th; $\pm y, \pm v$ are in the same column of $B_{1}$, say the $n$ th. Considering the tables which have determined the positions of the elements in $A_{1}$ and $B_{1}$, we obtain that $\pm x y$ and $\pm u v$ are summands of the $(m, n)$-entry of $A_{1} B_{1}$. But it is readily seen that " - " was adjoined to only one element of each equation. Hence if $x y=u v$ is an internal equation the contribution of $x y$ and $u v$ to the $(m, n)$-entry of $A_{1} B_{1}$ is $\pm(x y-u v)=0$. If $x y=u v$ is the external equation, one of the words $x y$ and $u v$ is $a_{1} b_{1}$ and the other is $e f$, and their contribution to the (1,s+1)-entry of $A_{1} B_{1}$ is $a_{1} b_{1}-e f=w$. Thus, the $(1, s+1)$-entry of $A_{1} B_{1}$ is $w$ and the other entries are 0 and we have:

$$
A_{1} B_{1}=\left(\begin{array}{ll}
0 & w \\
0_{r s} & 0
\end{array}\right)
$$

where $0_{r s}$ is the $r \times s$ zero matrix.

Now, let $A^{\prime}$ be defined as the $(s-1) \times(r+s)$ matrix

$$
t\left(E_{1, r+2}+E_{2, r+3}+\cdots+E_{s-1, r+s}\right)
$$

and $B^{\prime}$ as the $(r+s) \times(r-1)$ matrix $t\left(E_{11}+E_{22}+\cdots+E_{r-1, r-1}\right)$. Note that $A^{\prime}$ is defined for $s>1$ and $B^{\prime}$ is defined for $r>1$. We enlarge the matrices $A_{1}, B_{1}$ to matrices $A, B \in R_{l}$ by the aid of $A^{\prime}$ and $B^{\prime}$ as follows:

$$
A=\left(\begin{array}{c}
A^{\prime} \\
A_{1}
\end{array}\right), \quad B=\left(B_{1} \mid B^{\prime}\right)
$$

If $s=1$ we take $A=A_{1}$ and if $r=1$ we take $B=B_{1}$. We obtain

$$
\left.A B=(\underbrace{\underbrace{A^{\prime} B_{1}} \mid A^{\prime} B^{\prime}}_{s+1} \underbrace{A_{1} B_{1}}_{r-1} \mid \underbrace{A_{1} B^{\prime}}_{1})\right\} r+1
$$

and we have

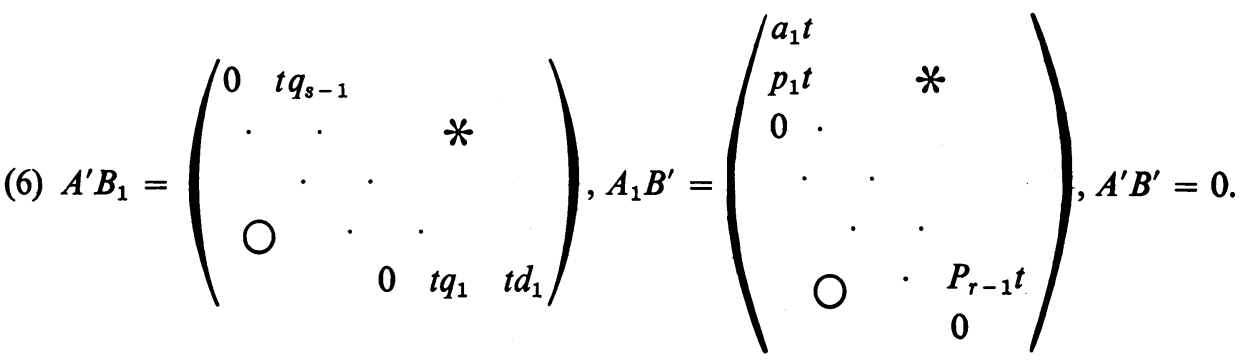


By (4), (5) and (6) we obtain

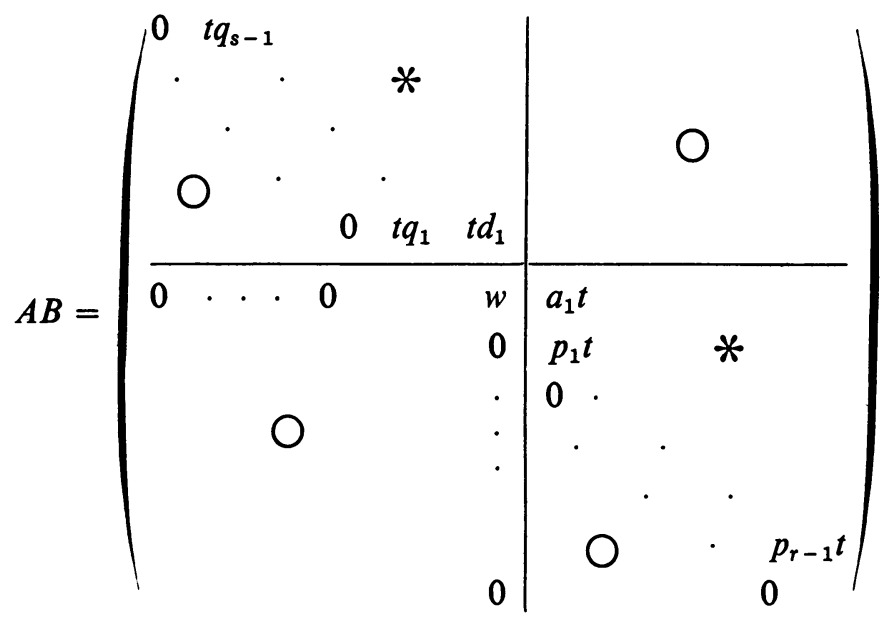

Now, we complete the proof of Theorem 7. We have $(A B)^{l}=0$ since $A B$ is triangular. Hence by the remark at the beginning of this section it follows that $(B A)^{l}=0$. We also see that

$$
(A B)^{l-1}=\left(t q_{s-1}\right) \cdots\left(t q_{1}\right) w\left(p_{1} t\right) \cdots\left(p_{r-1} t\right) E_{1 l} .
$$

The $(r+1,1)$-entry of $B$ is $q_{s}$ and the $(l, r)$-entry of $A$ is $p_{r}$. But $E_{r+1,1} E_{1 l} E_{l r}=E_{r+1, r}$ and therefore we obtain for the $(r+1, r)$-entry of $B(A B)^{l-1} A=(B A)^{l}=0$ the equation

$$
q_{s} t q_{s-1} \cdots t q_{1} w p_{1} t \cdots p_{r-1} t p_{r}=0 .
$$

Since $R$ is an integral domain and $p_{1}, \ldots, p_{r}, q_{1}, \ldots, q_{s}, t$ are nonzero elements it follows that $w=0$ and this completes the proof of the theorem.

In [4] we have constructed integral domains $R$ which do not satisfy $\mathfrak{R}$, but $R^{*}$ are embeddable in groups and therefore $R$ satisfy $\mathfrak{M}$. Hence by Theorem 7 we obtain

THEOREM 8. $\mathfrak{R} \varsubsetneqq \mathfrak{M}$.

5. The relation between $\mathfrak{R}_{l}$ and $\mathfrak{M}_{l}$. In this section we obtain the relation between the conditions $\mathfrak{N}_{l}$ and $\mathfrak{M}_{l}$ (defined in the introduction) for integral domains.

The conditions of $\mathrm{Mal}^{\prime} \mathrm{cev}$ of order 1 are satisfied by a cancellative semigroup and in particular they are satisfied by $R^{*}$ if $R$ is an integral domain. Thus, each integral domain satisfies $\mathfrak{M}_{1}$. Since an integral domain has no nonzero nilpotent elements it satisfies $\mathfrak{R}_{1}$ and this implies $\mathfrak{N}_{1}=\mathfrak{M}_{1}$.

To obtain the relation between $\mathfrak{R}_{l}$ and $\mathfrak{M}_{l}$ for $l \geqq 2$ we first prove

LEMMA 9. $\mathfrak{N}_{l} \supseteq \mathfrak{N}_{l+1}$. 
Proof. Let $R$ be an integral domain which does not satisfy $\mathfrak{N}_{l}$ and we shall prove that it does not satisfy $\mathfrak{N}_{l+1}$. Let $A \in R_{l}$ be nilpotent of order $n>l$. Thus, $A^{n}=0$, $A^{n-1} \neq 0$ and assume that the $(p, q)$-entry of $A^{n-1}$ is $a \neq 0$. Let $B_{1} \in R_{l+1}$ be the matrix obtained from $A$ by adjoining a row and a column of zeros and let $B=B_{1}$ $+t E_{a, l+1}$, where $0 \neq t \in R$. Computing the powers of $B$ we obtain $B^{n+1}=0$ and $B^{n} \neq 0$ since its $(q, l+1)$-entry is $a t \neq 0$. Thus, $B \in R_{l+1}$ is nilpotent of order $n+1$ $>l+1$, hence $R$ does not satisfy $\mathfrak{R}_{l+1}$.

Using the previous lemma and the result proved in Theorem 7, we obtain

LEMMA 10. $\mathfrak{N}_{l} \subseteq \mathfrak{M}_{l}$.

Proof. By the proof of Theorem 7 it follows that if $R$ satisfies $\mathfrak{N}_{l}$, then $R^{*}$ satisfies all the cancelled conditions of order $l$. But, by the previous lemma, $R$ satisfies $\mathfrak{R}_{k}$ for $k \leqq l$, hence $R^{*}$ satisfies all the cancelled conditions of order $\leqq l$. Now, each condition of order $l$ which is not cancelled can be proved using the cancellation laws and perhaps also a cancelled condition of lower order. This proves that $R^{*}$ satisfies all the conditions of order $l$ and hence $R$ satisfies $\mathfrak{M}_{l}$.

In [4] we have constructed for each $l \geqq 3$ an integral domain $R$ which does not satisfy $\mathfrak{N}_{l}$, and $R^{*}$ is embeddable in a group. Hence $R$ satisfies $\mathfrak{M}$ and in particular it satisfies $\mathfrak{M}_{l}$. This result together with the result of Lemma 10 implies

THEOREM 11. $\mathfrak{N}_{l} \subsetneq \mathfrak{M}_{l}$ for each $l \geqq 3$.

Now, we deal with the case $l=2$. We first prove

LEMMA 12. If $R$ is an integral domain and $A \in R_{2}$ is nilpotent of order $k+1, k \geqq 2$, then all the entries of $A$ and of $A^{k}$ are $\neq 0$.

Proof. Let $A=\left(a_{i j}\right)$ and $A^{k}=\left(b_{i j}\right)$. Since $A^{k} \neq 0$ and $k \geqq 2$ we have $A^{2} \neq 0$.

If $a_{12}=0$ then the $(1,1)$ and $(2,2)$ entries of $A^{k+1}$ are $a_{11}^{k+1}$ and $a_{22}^{k+1}$ respectively. But $A^{k+1}=0$ and since $R$ is an integral domain it follows $a_{11}=a_{22}=0$. Hence we obtain $A^{2}=0$, a contradiction. Thus, $a_{12} \neq 0$ and similarly $a_{21} \neq 0$.

If $a_{11}=0$ then since $A A^{k}=A^{k+1}=0$ we obtain $a_{12} b_{2 j}=a_{11} b_{1 j}+a_{12} b_{2 j}=0$ for $j=1,2$. Hence $b_{21}=b_{22}=0$ and since $A^{2 k}=0$ its $(1,1)$-entry is $b_{11}^{2}=0$ which implies that $b_{11}=0$. By the relation $A^{k} A=A^{k+1}=0$ we obtain $b_{12} a_{21}=b_{11} a_{11}+b_{12} a_{21}=0$ and therefore $b_{12}=0$. Thus, we have proved $A^{k}=0$, a contradiction. Hence $a_{11} \neq 0$ and similarly $a_{22} \neq 0$ and this completes the proof that all the entries of $A$ are $\neq 0$.

Now, we prove that all $b_{i j} \neq 0$. We have $a_{11} b_{1 j}+a_{12} b_{2 j}=0$ and since $a_{11}, a_{12} \neq 0$ it follows that $b_{1 j}=0$ if and only if $b_{2 j}=0$. Similarly the equation $b_{i 1} a_{11}+b_{i 2} a_{21}=0$ implies $b_{i 1}=0$ if and only if $b_{i 2}=0$. Hence if $b_{i j}=0$, then the second entry of the $j$ th column of $A^{k}$ is 0 , and therefore the second column of $A^{k}$ is also 0 , a contradiction. This proves that each $b_{i j} \neq 0$.

THEOREM 13. $\mathfrak{R}_{2}=\mathfrak{M}_{2}$. 
Proof. By Lemma 10 we have $\mathfrak{R}_{2} \subseteq \mathfrak{M}_{2}$. To obtain equality it suffices to prove that if $R$ is an integral domain which does not satisfy $\mathfrak{N}_{2}$, then it does not satisfy $\mathfrak{M}_{2}$.

Let $R$ be an integral domain and let $A \in R_{2}$ be nilpotent of order $k+1, k \geqq 2$. We shall show that $R$ does not satisfy $\mathfrak{M}_{2}$. Let us consider the following condition of $\mathrm{Mal}^{\prime}$ cev of order 2:

$$
a_{1} b_{1}\left({ }_{1} p_{1} b_{1}=c_{1} d_{1}\left[{ }_{1} c_{1} q_{1}=p_{1} b_{1}^{\prime}\right)_{1} a_{1} b_{1}^{\prime}=c_{1}^{\prime} q_{1}\right]_{1} c_{1}^{\prime} d_{1} .
$$

As in the previous lemma let $A=\left(a_{i j}\right), A^{k}=\left(b_{i j}\right)$. Since $A^{k} \neq 0$ and $k \geqq 2$ we have $A^{2} \neq 0$ and suppose the $(i, j)$-entry of $A^{2}$ is not 0 . We take:

$$
\begin{aligned}
& a_{1}=a_{i 1}, \quad p_{1}=b_{i 1}, \quad b_{1}=a_{1 j}, \quad b_{1}^{\prime}=b_{1 j} \\
& d_{1}=a_{2 j}, \quad q_{1}=b_{2 j}, \quad c_{1}=-b_{i 2}, \quad c_{1}^{\prime}=-a_{i 2} \text {. }
\end{aligned}
$$

By Lemma 12 all these elements are $\neq 0$ (belong to $R^{*}$ ) and since $A^{k} A=A A^{k}$ $=A^{k+1}=0$ and $A^{k} A^{k}=0$ we obtain

$$
\begin{aligned}
& p_{1} b_{1}=b_{i 1} a_{1 j}=-b_{i 2} a_{2 j}=c_{1} d_{1}, \\
& c_{1} q_{1}=-b_{i 2} b_{2 j}=b_{i 1} a_{1 j}=p_{1} b_{1}^{\prime}, \\
& a_{1} b_{1}^{\prime}=a_{i 1} b_{1 j}=-a_{i 2} b_{2 j}=c_{1}^{\prime} q_{1} .
\end{aligned}
$$

Thus, for 8 elements of $R^{*}$ the internal equations of (7) hold, but since the $(i, j)$ entry of $A^{2}$ is not 0 we have $a_{1} b_{1}=a_{i 1} a_{1 j} \neq-a_{i 2} a_{2 j}=c_{1}^{\prime} d_{1}$ which means that the external equation of (7) does not hold. Hence $R$ does not satisfy $\mathfrak{M}_{2}$ and this completes the proof of the theorem.

The following is an application to our main result which has been communicated to us by the referee $\left({ }^{2}\right)$.

A semifir (=local fir in [2]) is an integral domain in which every finitely generated left (or right) ideal is free of unique rank.

THEOREM 14. A semifir satisfies $\mathfrak{N}$ and hence its multiplicative semigroup is embeddable in a group.

Proof. Let $A$ be an $l \times l$ matrix over a semifir $R$ and assume $A^{n}=0$ for some $n$, but $A^{n-1} \neq 0$.

Write $V=R^{l}$ considered as left $R$-module; by [2, Proposition 2.1] all finitely generated submodules of $V$ are free. Moreover multiplication (on the right) by $A$ defines an $R$-homomorphism. Thus writing $V_{i}=V A^{i}$ we have the chain of submodules

$$
V=V_{0} \supseteq V_{1} \supseteq \cdots \supseteq V_{n-1} \supseteq V_{n}=0 .
$$

All these submodules are free of diminishing ranks, hence if $n>l$ we have equality at some stage, say rank $\left(V_{i-1}\right)=\operatorname{rank}\left(V_{i}\right)$. But this means that $A$ acting on $V_{i-1}$ is injective, whence so is $A^{n}=0$, i.e. $V_{i-1}=0$, a contradiction unless $n \leqq l$.

$\left.{ }^{2}\right)$ The author wishes to thank the referee for this interesting application. 
We conclude with the following remark.

A ring $R$ satisfying $\mathfrak{R}$ has the following property for every integer $n$ : If $A, B \in R_{n}$ and $A B=1$ then $B A=1$. Indeed, if $A B=1$ and $B A \neq 1$ then as in [5, p. 35] define $e_{i j}=B^{i-1} A^{j-1}-B^{i} A^{j}$ for $i, j=1,2, \ldots$, and observe that for each $m \geqq 2, e_{12}+e_{23}$ $+\cdots+e_{m-1, m}$ is nilpotent of order $m$, a contradiction.

In [3] the property " $A, B \in R_{n}$ and $A B=1$ then $B A=1$ " is expressed in the following equivalent form:

III. In a free $R$-module of any rank $n$, any generating set of $n$ elements is free. Hence a ring satisfying $\mathfrak{N}$ has the property III and also satisfies II and I of [3] and in particular it has the invariant basis property.

\section{REFERENCES}

1. P. M. Cohn, Universal algebra, Harper \& Row, New York, 1965.

2. - Free ideal rings, J. Algebra 1 (1964), 47-69.

3. - Some remarks on the invariant basis property, Topology 5 (1966), 215-228.

4. A. A. Klein, Rings nonembeddable in fields with multiplicative semigroups embeddable in groups, J. Algebra 7 (1967), 100-125.

5. N. Jacobson, Structure of rings, Amer. Math. Soc. Colloq. Publ., Vol. 37, Amer. Math. Soc., Providence, R. I., 1964.

6. A. I. Mal'cev, On the immersion of an algebraic ring into a field, Math. Ann. 113 (1937), 686-691.

7. —_ Uber die Einbettung von assoziativen Systemen in Gruppen, Math. Sb. 6 (1939), 331-336.

Tel-Aviv University,

Tel-Aviv, IsRael 